of the patient was so critical, that a thoroughly satisfactory curative procedure could not be undertaken ; therefore the gap was simply closed with a series of five or six stout silk sutures. Each of these included the peritoneum and the whole thickness of the abdominal wall, and the lowest were passed so as to pleat up and obliterate the neck of the sac. As suppuration is apt to occur in these cases it is, perhaps, better not to bury these sutures, but to bring their ends out through the skin in the usual way ; also stout silkworm gut may be advantageously substituted for silk.

The ultimate results of this somewhat crude operation have been better than I expected. One case was very instructive. The patient had a large oblique inguinal hernia upon the right side, which had been strangulated in 1880 and operated upon. No radical cure was effected, and it came down again in spite of a truss. I operated for strangulation on July 1st, 1886, and effected a radical cure. I saw this man on Sept. 2nd, 1893, with a sound abdominal wall, although in the interval he had worked hard and had been spared the inconvenience of a truss. Another man, who had an enormous hernia, said a year after the operation that he was cured and wore no instrument, being able to work as a cabman; but this case ultimately relapsed.

Like other surgeons who practise in London, I have the greatest difficulty in tracing cases; therefore I am only able to illustrate the ultimate results of the operation of radical cure by giving such samples as I have been able to obtain. I claim, however, that they show that the operation of radical cure of inguinal and of femoral hernia is, when efficiently done upon proper cases, as successful as any operation in surgery. A young man aged twenty-two years was operated on on March 16th, 1892, for the cure of a right inguinal hernia. He wished to enter a trade society which would not admit him if he merely wore a truss. On Oct. 20th last his cure was perfect, he could do the hardest work, and had been a member of his trade society for a year. A gentleman who was operated on on April 21st, 1892, was quite well in October last, being able to shoot, ride, row, or do anything he wished. A schoolboy who was operated on on July $17 \mathrm{th}$ 1892, was quite well on Oct. 20th last, and is now able to play cricket and football. I have no cases of Bassini's operation of more than a year's standing, but I have had no relapse in this class, and those who have come up for inspection seem to be perfect. The ultimate results of the operation for the radical cure of femoral hernia have been quite satisfactory, although I can record a relapse in a case in which the neck of the sac was merely tied. One which was performed on a delicate woman fifty years of age, after strangulation, on Jan. 5 th, 1889, showed no tendency to relapse on May 14th, 1890, and she required no instrument until her death, which occurred in the following year. Mr. H. Fraser Stokes reports that a cook aged forty-eight years, who was operated on for right and left femoral epiploceles on Jan. 25th, 1891, was quite well on Oct. 20th, 1893. Also that one of her fellow servants, who bad a small irreducible femoral epiplocele, wished to have the same operation performed. It: would not be easy to find more striking evidence than this of the benefits of the procedure. I have only met with two cases of relapse, although I have taken great pains to keep the cases under observation. In one case the relapse was due to suppuration; it occurred six months after the operation. The patient elected to wear a truss, which he could not do before. The second relapse was due to a faulty operation. I performed the ordinary one on a young man, and six months afterwards his hernia recurred while he was lifting a heavy weight. As the first operation had caused him no pain and very little inconvenience he came back to ask to have it carried out again. As the relapse was very small and high up the inguinal canal the aponeurosis was incised and Bassini's operation was performed. This enabled me to ascertain that the relapse was due to about three-quarters of an inch of sac having been left behind on the first occasion.

Upper Berkeley-street, w.

Iondon Post-Graduate Course.-The vacation courses in Bacteriology, Pathology and Hygiene, have been arranged and will commence on Monday, Dec. 4th, and end on Friday, Dec. 15th. The gratifying success of the September vacation classes shows that there are many practitioners who can only leave their work for ten or eleven days at a time and are anxious to receive further instruction in these important subjects. The class lists are now being made nut, and entries may be made on application to Dr. Fletcher Little, 32, Harley-street, W.
THE

DETACHMENT OF THE EPIPHYSIS FOR THE ANTERIOR INFERIOR SPINOUS PROCESS OF THE ILIUM THROUGH ENFORCED MUSCULAR ACTION.

BY R. HENRY ANGLIN WHITELOCKE, M.B., M.C. EDIN., F.R.C.S. ENG.

SURGICAL literature affords but few examples of the condition which the above heading describes. With the single exception of a case mentioned by Mr. F. Treves, ${ }^{1}$ in which it occurred while the subject was in "the act of running a race," examples are not to hand. For this, among other reasons, it has been considered to be advisable to place on record the clinical details of two cases which have lately occurred in my practice. The hope is also entertained that by so doing cases which may have been observed by others will now be recorded. My belief is that this accident is probably not so rare as at first would seem to be the case. Besides entering inte the clinical details of the cases it is proposed to more fully consider and inquire into the anatomy and etiology of the injury as well as to deal with its treatment. The clinical histories and symptoms of the two cases are so nearly identical that one description might almost suffice for both. One evening early in February, 1891, an undergraduate drove up to my house, and after hobbling into my room gave the following bistory. In starting to run in a race of 100 yards distance, while practising for his college sports, he "seemed to have scarcely travelled ten yards when he had suddenly to stop as he felt something snap, and at the same time his limb became powerless." There was then some pain, but it was " by no means severe," although he " became faint and felt sick." On removing his clothes there was little to be seen excepting that the injured limb assumed the position of semiflexion and was in advance of its fellow, which, being fully extended, was sustaining the whole weight of the patient. When asked to straighten the injured limb by extending the hip- and knee-joints increased pain was felt, and he had extreme difficulty in extending the knee-joint; but what he most complained of was an unpleasant feeling of numbness and tingling perceived all down the extensor surface of the thigh. There was very little discolouration, accompanied by an inconsiderable swelling situated for the most part beneath and to the outer side of the middle of Poupart's ligamentthe region, in short, of the anterior inferior spinous process of the ilium. After carefully comparing the two sides and palpating, a distinct crepitation could be elicited on deep pressure in the region of the swelling: and this could be even more distinctly made out when, with the hands placed over the seat of the injury, the patient made an effort to bring the rectus muscle into action. The morable piece of bone gave one a feeling on palpation not unlike that of a loose cartilage in a joint. The diagnosis was then made, but not before all the usual sites had been carefully examined for a possible her nia the history of something having snapped, the pain, and the feeling of sickness and faintness favouring such an occurrence. A pad with a temporary spica bandage was applied, and the patient was sent back to his college. On visiting him next morning the swelling had somewhat increased, but the discolouration remained about the same. There was pain only on movement, while the tingling sensations persisted unaltered. 'Two days after this another undergraduate from a different college was seen, and he gave an almost similar history. $\mathrm{He}$ had been starting to run "a quarter mile." In the very act of starting - in the first bound, as it were-he "felt something give, and became powerless to proceed." With the experience of the former case it was not difficult to discover what was the matter. In this case a mere scale of bone, as far as could be elicited, came away with the straight head of the rectus, and crepitus was with difficulty made out at first. The tingling sensation and other symptoms described in the former case were also evident.

Thus, the histories and causation of these cases were remarkably alike. Kach patient had started with his left limb somewhat flexed both at the hip- and knee-joints, and in advance of the right, the body being bent forwards and, as it were, 
poised upon and supported by it. In the sudden act of starting, the limb behind was used to give the spring, and in the first bound the body and the advanced and flexed limb being suddenly and quickly straightened together, the rectus muscle, already contracting and fixed at its lower attachment to support the weight of the body on the semi-flexed knee, being forcibly put upon the stretch during the sudden extension of the hip-joint, tore away the unossified epiphysis of the anterior inferior spinous process of the ilium. The respective ages of the patients were eighteen and nineteen years. If we for a few moments turn to the consideration of the anatomy of the parts here concerned in their bealthy condition it is not difficult to understand the pathology of this injury, and a question which almost at once presents itself to us is, Why is it that we do not more frequently meet with it? The parts most immediately concermed are the rectus femoris muscle, the anterior inferior spinous process of the ilium, and the ilio-femoral ligament. Briefly, according to Mr. Roger Williams, ${ }^{2}$ the rectus muscle is attached above solely by its acetabular posterior primary or reflected head until about the third month of fœetal life "for up to this time the flexed position of the foetal limb necessitates only this head, which in this position of the hip-joint is in a direct line of action." The iliac anterior or straight head is of secondary formation, and is developed from the sheath of the posterior tendon "owing to the change

Fig. 1.

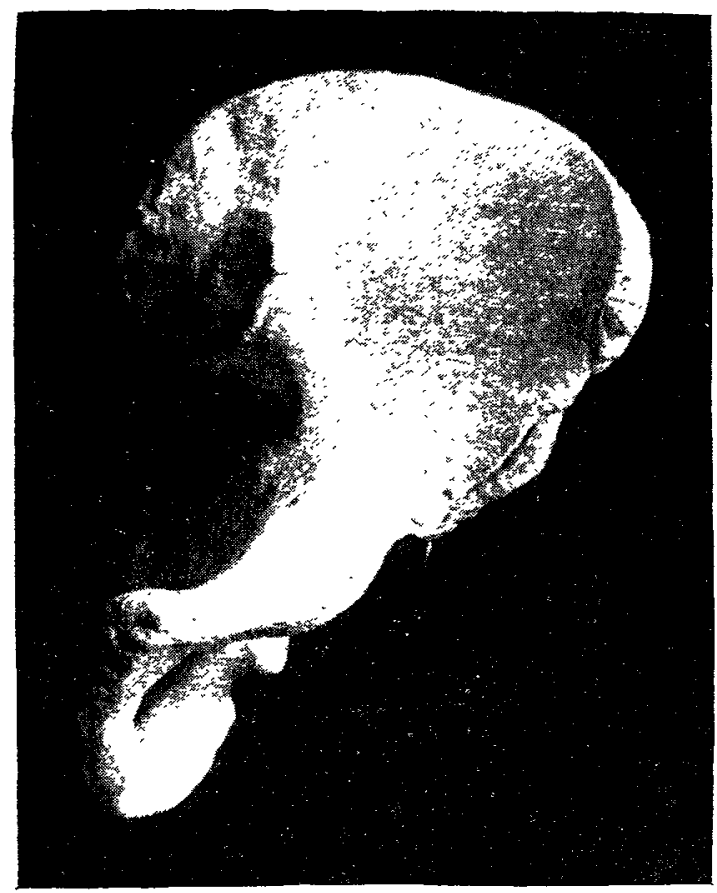

of axis of the limb consequent on the assumption of the capacity of full extension of the hip and knee." 3 The muscle acts chiefly from its straight head when the hip is extended, but from its curved head when it extends the knee during flexion of the hip. Thus in the position which was described by the patients as obtaining prior to starting - viz., that of flexion of the hip and semiflexion of the knee-the posterior head would fix the rectus above; but in the first bound of the start, when the knee-joint and hip-joint were suddenly, rapidly, and forcibly extended, the direct or straight head would come into action. This direct head, though it has been shown conclusively by Mr. Roger Williams to be developed secondarily, and to be of different histological structure (partaking more of the nature of a ligament than of a tendon) from its neighbour, is exceedingly strong in well-developed athletic and muscular subjects. The anterior inferior spine of the ilium is peculiar to the human pelvis. It is hardly to be $n$ oticed as a prominence in the higher apes. In the mammalia generally, according to Professor Flower, " "the acetabular border of the innominate bone is very short and has a strong ${ }^{2}$ Anatomy of the Quadriceps Extensor, Journal of Anatomy and
Physiology, vol. $i$., 187y.

4 : Text-book of Human Anatnmy, p. 488

4 Osteology of the Mammalia, p. $3 \pm t$. rounded rough prominence for the attachment of the rectus (extensor) muscle of the leg." In the human pelvis "it serves to give a slight leverage to the rectus femoris, making some amends to that muscle for the unfavourable position in which it is placed by the erect posture." 5 The anterior inferior spinous process of the ilium is in the adult (as may be well seen in the accompanying engraving, which is from a photograph) made up by the ossification of a secondary epiphysis for the innominate bone, which makes its appearance in the fifteenth or sixteenth year, and which is consolidated at the nineteenth or twentieth year.

The accompanying figures of a macerated bone from a subject aged sixteen years were engraved from photographs. They show well the prominence, large size, and loose connexion of this epiphysis at this period. The anterior superior spine has not a distinct epiphysis for itself, but is ossified from the large secondary one common to it and the whole of the iliac crest. The ilio-femoral ligament is attached above to the inferior spine, below, blending with the capsular ligament of the hip-joint to the femur. It is obvious, therefore, that in the separation of this process the hip-joint may be affected and thus explain the discomfort which existed in extending these injured limbs, as well as the flexed position which was assumed. The nerve-supply to the muscle is the anterior crural, which gives a branch to enter its upper part posteriorly. The arterial supply originates in the external circumflex and

FIG. 2.

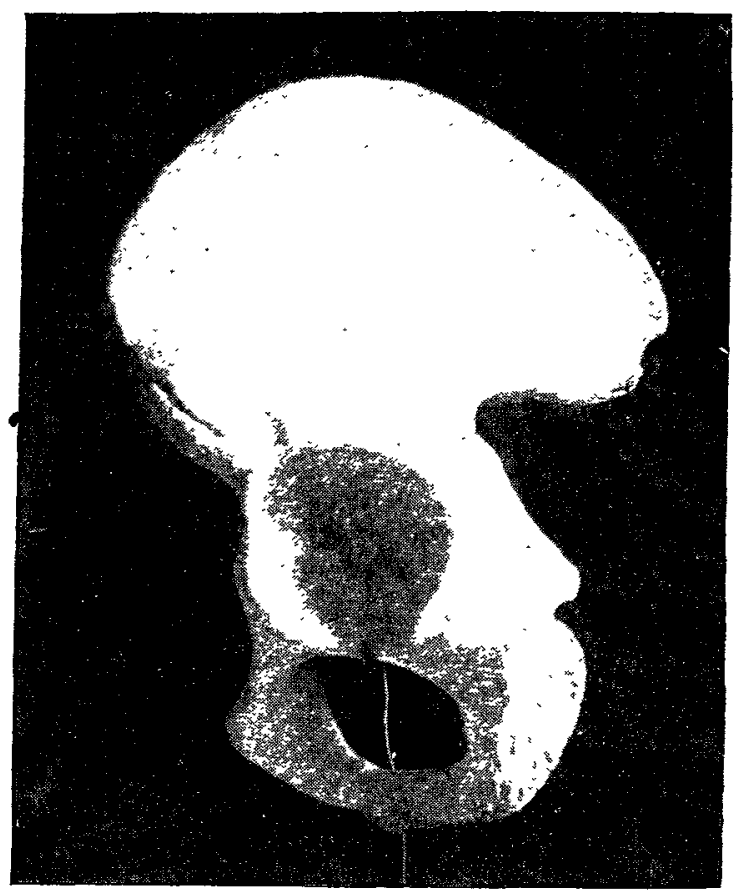

the femoral vessels for the most part. It will thus be seen. that the above anatomical details are not here irrelevant, for the whole pathology of the injury is evident on referring to them. In these young athletic men of good muscular development, with powerful recti muscles and at the ages when the epiphyses for the spinous processes were as yet unconsolidated and thereby able to be detached, is it surprising that under the unusual and sudden strain placed upon these parts that an accident did occur? The wonder is that we do not more frequently meet with these cases among athletes. Possibly the explanation may be in the fact that "sprinters" as a rule get into better athletic training before they begin to practise "starting." In these cases the occurrence took place at the beginning of term and before the men were in proper training. The tingling feeling described in each case may be explained by a displacement of the rectus muscle in the upper part of its course, with stretching of the fascia lata of the thigh and a disturbance in the cutaneous. supply of the anterior crural nerve. The comparatively small amount of swelling and bruising may be accounted for by theanatomy of the region, which is not very vascular and is well supported by muscles and the thick fascia of the thigh. This 
fascia would also naturally tend to conceal an extravasation beneath it.

Remarks. - The diagnosis of these cases is by no means as easy as might be expected from this description. The history at once demanded the exclusion of the possibility of an existing hernia, and the posture of the limb suggested forcibly at first an injury to the hip-joint. The crepitation, owing to the very limited displacement allowed by the indirect head of the rectus, the fascial attachments of the parts, and the deep position of the fracture in a muscular limb, is not sasily brought out. In this relationship also I would suggest that, arguing from anatomical grounds, the statements made in many of our text-books in a somewhat vague manner that "separation of both the upper and lower anterior spinous processes of the ilia occurs through muscular violence" require corroboration. The anterior superior process develops, as before stated, from the epiphysis for the whole of the iliac crest and would, therefore, require even more violence to effect its separation. Again, the sartorius muscle would seem to be neither sufficiently strong in itself nor sufficiently concentrated in its attachments to do much harm, nor is it easy to conceive of any of its actions which would pull sufficiently forcibly upon this process; and so it would appear that many of the so-called separations of the anterior superior process may have been in reality that of the above-described condition. The two cases were reated after the same manner. A plaster spica bandage, well padded, was applied, the limb being kept in a position adapted to relax the direct head of the muscle. This was retained for four weeks and then a simple spica bandage was substituted. The patients did well, and gave no trouble. The "tingling and feeling of cold water" were the most peristent symptoms in one case, and in this case a consiclerable amount of callus was thrown out, but notwithstanding this the patient is now able to run successfully. The other patient has "given up sprinting, as he has lost his pace." Probably in men who start in races and hold their bodies more erectly than these did the risk is not so great. By a strange coincidence, during the same week that these cases presented themselves another patient was seen at another college by Mr. Symonds and Dr. Ritchie of this city, also the result of running in a race. This was treated in much the same way, and with a satisfactory result. One might with equal propriety and probably with as little scientific spirit suggest the term of "sprinter's fracture" for this injury as those who have given expression to "rider's sprain," "tennis leg," \&c., in surgical literature.

oxford.

\section{ON THE THERAPEUTIC USES OF EXALGINE.}

BY THOMAS D. SAVILL, M.D. LOND.,

FORMERLY MEDICAL SUPERINTENDENT OF THE PADDINGTON INFIRMARY.

MY attention was called to this new analgesic by the various favourable reports that were published from time to time in the medical journals, and I decided to employ it in a -ertain number of suitable cases, of which there is usually no lack in an institution like the Paddington Infirmary. I have ased it, not so much as a curative agent in neuralgia and allied disorders of the nervous system, as in the endeavour to relieve the pain associated with various chronic maladies. An agent which will enable the medical man to relieve even where he cannot hope to cure is necessarily one of great value, and the mitigation of pain is a thing which the patient looks to him to effect perhaps even more than the ultimate cure of the affection. Detailed notes of the various cases were kept, the particulars of which I am enabled to summarise hereafter, thanks to the kind assistance of Mr. Harold V. Prynne, who was my clinical assistant at the time.

It is hardly necessary here to dwell upon the physical eharacters and physiological properties of the drug; it will suffice to say that it can be readily administered in the form of a powder, especially if put up in "cachets," or it may be dissolved in water to which a small proportion of spirit has been added, or even in pure water if this be wsed hot. In fact, the last-mentioned method of administration is, on the whole, the most convenient one, for if six suid ounces of boiling water are poured on to forty-eight grains of the crystals a clear solution containing eight grains to the fluid ounce is formed, which does not precipitate on cooling; a teaspoonful of this solution thus represents one grain of the dry salt. This keeps well, is devoid of disagreeabie smell or taste, and is in every way reliable. It will be seen that the diseases for which the patients were under treatment were various; and, speaking generally, it may be affirmed that in no instance did the drug give rise to any inconvenient or uncomfortable effects even in the presence of cardiac disease or phthisis, nor did it determine any of the symptoms of intoxication which seem to have been manifested in the hands of other observers. These symptoms may perhaps be attributed to an overdose or to some idiosyncrasy on the part of the patient; and I think that, if care is taken not to administer the drug to patients with a febrile temperature or who are constipated, no ill-effects are ever likely to be experienced. These are, at any rate, the conclusions which I have drawn from $m y$ perhaps somewhat limited experience.

CASE 1.-A woman aged fifty-eight years, suffering from carcinoma uteri, had severe attacks of abdowinal pain of a dull, continuous character, interfering with rest and nutrition. She was given two grains every two or three hours. No effect was produced on the sleeplessness, but the relief to the pain was immediate and marked. Later it was found to be necessary to increase the dose to three grains, but no tolerance seemed to be established towards the drug, and relief was afforded as long as it was persisted in. No giddiness or other disagreeable after-effect was ever complained of.

CASE 2.-A woman aged twenty-eight years suffered from neurasthenic cephalalgla of long standing, the pain being dull, severe, and continuous. She was given one-grain doses three times a day, immediate relief being afforded to the headache. Sleep, which had previously been disturbed and uncertain, became calm and refreshing, and she was soon convalescent.

CASE 3. - The patient in this case was a woman aged seventy-four years, who suffered from rheumatic arthritis. She complained of severe neuralgic pains in the head and face, with a tendency to radiate in various directions. I gave her two grains of exalgine every hour, and she experienced marked relief within a short time of the first dose. It was subsequently found to be necessary to increase the dose in order to maintain the effect, but, this having been done, her condition was markedly ameliorated.

CASE 4.-A woman aged sixty-three years, suffering from cardiac valvular disease, had complained of intense paroxysms of pain over the brow ; this was of a continuous "stabbing" character, subject to periodical exacerbations. Sleep was not interfered with, as a rule. She was given one grain three times a day and was speedily relieved of this particular manifestation.

CASE 5.-This case was one of chronic phthisis associated with multiple neuritis together with neuralgic manifestations specially affecting the head and eyes ; the pain was continuous, but, as in the preceding case, subject to periodical exacerbations. She was ordered two grains of the drug every two hours, but though the suffering became less acute-that is to say, the exacerbations were apparently prevented-complete relief was not obtained even on pushing the remedy. This patient suffered from sleeplessness, and a marked improvement was observed in this respect.

CASE 6 -This was another case of cardiac valoular disease, occurring in a woman aged forty-two years, who suffered a great deal from periodical attacks of shooting pain in the head and neck. The administration of occasional doses of two grains of exalgine effectually relieved the attacks as they occurred, and this within a very short space of time.

CASE 7 was an interesting case of traumatic epilepsy in a man aged forty-four years. He had undergone cerebrectomy for the relief of the epilepsy, after which he suffered from spastic hemiplegia associated with sensory manifestations chiefly in the head, right knee, and right hand, of a lancinating character. The pain effectually prevented sleep, and the patient was in a most miserable condition. I ordered him one-grain (subsequently increased to twograin) doses of exalgine, to be taken every four or five hours; after the first two doses he experienced considerable relief, the attacks of pain being less frequent and less severe. I did not, however, succeed in abolishing the painful manifestations altogether. The insomnia was improved while he was taking the drug, doubtless by reason of the relief afforded to the painful symptoms. This patient complained of slight headache after taking the drug, but it is doubtful how far 